Preprint typeset in JHEP style - HYPER VERSION

\title{
On the quantum width of a black hole horizon
}

\author{
Donald Marolf \\ Physics Department, UCSB, Santa Barbara, CA 93106. marolf@physics.ucsb.edu
}

\begin{abstract}
The many low energy modes near a black hole horizon give the thermal atmosphere a divergent entropy which becomes of order $A / 4 G$ with a Planck scale cut-off. However, Sorkin has given a Newtonian argument for $3+1$ Schwarzschild black holes to the effect that fluctuations of such modes provide the horizon with a non-zero quantum mechanical width. This width then effectively enforces a cut-off at much larger distances so that the entropy of the thermal atmosphere is negligible in comparison with $A / 4 G$ for large black holes. We generalize and improve this result by giving a relativistic argument valid for any spherical black hole in any dimension. The result is again a cut-off $L_{c}$ at a geometric mean of the Planck scale and the black hole radius; in particular, $L_{c}^{d} \sim \frac{R}{T_{H}} \ell_{p}^{d-2}$. With this cut-off, the entropy of the thermal atmosphere is again parametrically small in comparison with the Bekenstein-Hawking entropy of the black hole. The effect of a large number $N$ of fundamental fields and the discrepancies from naive predictions of a stretched horizon model are also discussed. For the proceedings of the Adriatic 2003 conference.
\end{abstract}




\section{Contents}

1. Introduction 1

2. Describing Near-horizon degrees of freedom 3

3. Estimating the width

4. Discussion 8

A. An overly conservative estimate 11

\section{Introduction}

The work presented by the author at Adriatic 2003 comments on arguments [1], 2, 3, 4] which purport to derive fundamental bounds on the entropy of any system from the so-called generalized second law of thermodynamics; i.e. the second law including the BekensteinHawking entropy $S_{B H}=A / 4 G$ for black holes. A number of interesting loopholes were pointed out stemming from the effect of the thermal atmosphere (see [5, 6]) and an additional "observer dependence" in the concept of entropy (see [7]). However, this work is well described in [5, 6] and [7]. Thus, rather than repeat the story in detail, it seems best to use this proceedings to discuss other thoughts on black hole entropy that are only vaguely related to the material presented at the conference. Nonetheless, a brief summary of that material will be included in the discussion below where it ties in to the current storyline (section 4).

The main focus here will be on the many low energy degrees of freedom near the horizon of a black hole. Our primary interest will be in non-degenerate (i.e., non-extremal) horizons, with the degenerate case being treated only as a limit. Such degrees of freedom give rise to an old puzzle reviewed in [8]. The puzzle is that, when described using quantum field theory in a fixed curved background spacetime, such degrees of freedom give a divergent contribution to the entropy of the black hole's thermal atmosphere ${ }^{1}$. If cut-off at the Planck scale, the thermal atmosphere naturally yields [9] an entropy of order the BekensteinHawking entropy $A / 4 G$ and potentially provides a large correction to the entropy of the black hole. This is closely related (see e.g. [10] and [11]) to the suggestion [12] that the one should consider the entanglement entropy of the quantum fields outside the black hole. In [9], it was in fact suggested that this calculation might in fact account for the entire

\footnotetext{
${ }^{1}$ i.e., the thermal bath of radiation constantly being emitted and re-absorbed by the black hole due to the Hawking effect.
} 
entropy of the black hole, though this idea appears to suffer from the so-called "species problem" and other issues discussed in [8].

Some time ago, it was argued by Sorkin [13] that one should cut-off the entropy of the thermal atmosphere at an even larger distance from the black hole horizon. Sorkin used a Newtonian model of a $3+1$ Schwarzschild black hole to argue that quantum fluctuations naturally cause the horizon to fluctuate on a scale $L_{c} \sim\left(R \ell_{p}^{2}\right)^{1 / 3}$ where $R$ is the black hole radius and $\ell_{p}$ is the Planck scale. Since the fluctuations are quantum in nature, we find it natural to describe this as providing a quantum "width" to the horizon. Clearly then, one cannot reliably characterize the region within $L_{c}$ of the classical horizon location as "outside" the black hole and it is reasonable to suppose a cut-off at this scale on contributions to the entropy of the (external) thermal atmosphere. Quantum fluctuations within $L_{c}$ of the classical horizon are then perhaps better described as fluctuations of the black hole itself, and may plausibly be assumed to already be included in the Bekenstein-Hawking entropy of the black hole. (Though of course the details of how or whether the full entropy is reflected in a spacetime description remains unclear.)

Since the entropy of the atmosphere scales with $A / L_{c}^{2}$, Sorkin's cut-off would make this contribution a parametrically small correction to the Bekenstein-Hawking entropy of a large black hole. However, one may ask to what extent Sorkin's Newtonian model captures the relevant relativistic physics, and one may also wonder whether a similar result is obtained for black holes with charge or in different numbers of dimensions. In particular, Sorkin's Schwarzschild correction is large enough that, if the same cut-off applied to the extremal case $^{2}$, one might expect it to have been seen in stringy studies [14] of sub-leading corrections to the Bekenstein-Hawking result for supersymmetric black holes.

We report here on work to clarify these issues. A general relativistic estimate is provided for the quantum width of the horizon for arbitrary spherical black holes. The result in $d$ spacetime dimensions is

$$
L_{c}^{d} \sim \frac{\ell_{p}^{d-2} R N^{1 / 2}}{T_{H}}
$$

where $N$ is the number of effective free fields propagating near the black hole and $T_{H}$ is the black hole's Hawking temperature. Again we see that $A / L_{c}^{d-1}$ is parametrically small in comparison with the Bekenstein-Hawking entropy, with the additional feature that the correction also becomes parametrically small ${ }^{3}$ as one approaches extremality and $T_{H} \rightarrow 0$. This is then in agreement with the results of [14], which finds no contributions clearly associated with the thermal atmosphere of a certain set of nearly extreme black holes. The full correction $N A / L_{c}^{2}$ can become large only when the number of fields $N$ becomes parametrically large. It is amusing to note that for Schwarzschild black holes in $3+1$

\footnotetext{
${ }^{2}$ Viewed as entropy of the thermal atmosphere, one would expect the entropy correction to vanish as $T_{H} \rightarrow 0$, but this is less clear when viewed as an entropy of entanglement across the horizon.

${ }^{3}$ The divergence of $L_{c}$ is unphysical and signals a breakdown of the near-horizon approximation used below, but nevertheless $L_{c}$ is much larger for a low $T_{H}$ black hole than for a black hole of similar size with $T_{H} \sim 1 / R$.
} 
dimensions $L_{c}$ is only just below nuclear length scales for astrophysical black holes and begins to approach atomic length scales for the largest known supermassive black holes.

These ideas and estimates are explained below. We begin in section 2 with a convenient description of the near-horizon degrees of freedom. We then perform the estimate in section 3. which is based on a certain reasonable conjecture with regard to distortions of the black hole horizon by nearby objects. We then discuss a few consequences and compare with the natural expectation based on a "stretched horizon" in section \&. Finally, we include an appendix which provides an estimate which does not rely on the above-mentioned conjecture, but which is clearly overly conservative. Nevertheless, this conservative argument yields qualitatively similar results in $d<6$ spacetime dimensions.

\section{Describing Near-horizon degrees of freedom}

In flat spacetime, any mode of any field which occupies a small region of space must have a correspondingly large energy. However, the diverging redshift at a horizon in principle allows for localized degrees of freedom with significantly smaller Killing energies. A particularly well-known modern application of this feature in the context of extremal horizons is the motivation [16] of the famous AdS/CFT conjecture. It will be useful for our discussion to study the near-horizon modes quantitatively. We give a simple description below and refer the reader to, e.g., 15] for a more technical treatment using tortoise $\left(r^{*}\right)$ coordinates.

Let us in particular consider a wavepacket localized within a proper distance $L$ of the horizon, where $L$ is much smaller than the local curvature scale. We focus on the nonextremal case so that, if the wavepacket is also localized on a scale $L$ in the directions along the horizon, the situation is well approximated by a wavepacket near a Rindler horizon in flat space.

Low energy such wavepackets will have essentially no variation on length scales shorter than $L$, except perhaps at proper distances $s \ll L$ from the horizon where we are free to take the wavepacket to vanish. Thus, they will look much like the larger wavepacket shown in figure 1 below. Since the energy density of the wavepacket is redshifted to zero near the horizon, the dominant contribution to the energy of the wavepacket will come from the part a proper distance $s \sim L$ away. Thus, the total energy of the wavepacket is of order $(L z)^{-1}$, where $z$ is the redshift at $s \sim L$ measured relative to infinity. A simple way to obtain this redshift for arbitrary black holes is to note that the local Unruh temperature at $s \sim L$ is of order $1 / L$, but it must also be related through the redshift to the Hawking temperature $T_{H}$ of the black hole. Thus, $(L z)^{-1} \sim T_{H}$, and the wavepacket in question represents an excitation with energy of order $T_{H}$.

Note that we obtain such a wavepacket for each $L$, so that there are an infinite number of modes with energy $T_{H}$ near any non-degenerate black hole horizon. But the thermal atmosphere of the black hole is just this same system at temperature $T_{H}$. It is therefore clear that such modes contribute an infinite entropy. In more detail, consider the number of such modes contained between surfaces a distance $L$ and $L+d L$ from the horizon (for $L$ small compared to the curvature scale). Since we considered wavepackets of size $L$ in each 


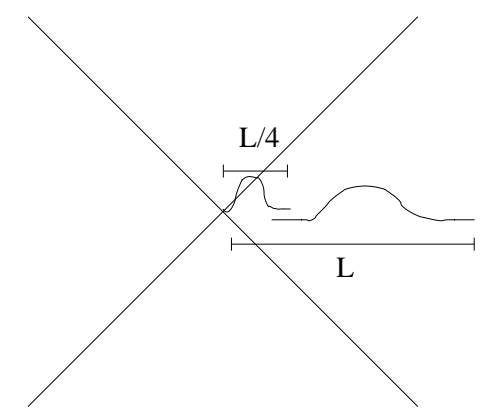

Figure 1: Two orthogonal low energy modes near a horizon. The larger has proper size $\sim L$ while the smaller is $\sim L / 4$. Both have energy $\sim T_{H}$.

direction, it is useful to take each surface (which has area $A$ ) and divide it into $A / L^{2}$ cells of size $L^{2}$.

In fact, a standard calculation using tortoise $\left(r^{*}\right)$ coordinates shows that a careful choice of wavepacket shape can result in arbitrarily low energies, say $\epsilon T_{H}$ for $\epsilon \ll 1$, within a proper distance $L$ of the horizon. However, such modes cannot be localized as well on the sphere, so that there are only $\lambda A / L^{2}$ of them and they contribute only $\mathcal{O}(1)$ changes in coefficients.

Finally, note that in the Rindler approximation a given mode is transformed into the next mode closer to the horizon by scaling it toward the bifucation surface (see figure 1 again). The relevant measure in the radial direction is therefore scale invariant and must be of order $d L / L$. (This result also follows from the logorithmic relation between the tortoise coordinate $\left(r^{*}\right)$ and proper distance 15].) Thus, the total number of modes between $L$ and $L+d L$ is of order $\frac{A}{L^{3}} d L$. Integrating the total contribution down to some ultraviolet cutoff $L_{U V}$ yields a total contribution of order $A / L_{U V}^{2}$. Each mode with energy $\lesssim T_{H}$ contributes a few bits of entropy, while modes with higher energy do not contribute. This then is the origin of the observation [9] that a Planck scale cutoff yields an entropy of order the Bekenstein-Hawking entropy of the black hole itself. It can also be mapped (see e.g. [10, 11]) to the corresponding result [12] for entanglement entropy.

\section{Estimating the width}

The proper interpretation of the thermal atmosphere's entropy is clearly an important issue in black hole thermodynamics. For example, in the most naive interpretation one might expect this to be a next-order correction to the Bekenstein-Hawking entropy $S_{B H}$. Unfortunately, with a Planck scale cut-off it is of comparable size to the "zero order" contribution $S_{B H}$. One may also try to cancel this entropy with a renormalization effect (though this creates puzzles when various objects are lowered toward the black hole horizon [8]), or to interpret the entropy of the thermal atmosphere as some sort of "dual" description of the Bekenstein-Hawking entropy itself [9, 12]. Unforunately, the latter approach suffers from the well-known "species problem" (i.e., it appears to depend on the number of propagating fields near the black hole horizon) and other concerns discussed in [8]. 
When the issue is phrased in terms of the near horizon modes, Sorkin's potential resolution suggests itself immediately. The presence of an extra particle with fixed energy too close to the horizon will effectively increase the mass of the black hole and will cause the horizon to expand outward and engulf the particle. If this particle is a quantum fluctuation associated with Hawking radiation, then it is natural to describe the effect as providing a non-zero quantum width for the horizon itself.

Consider for simplicity a $3+1$ Schwarzschild black hole and let $r$ be its Schwarzschild area-radius coordinate. The large entropy of the thermal atmosphere comes from modes near the horizon with energy $T_{H}$. But it is inconsistent to describe a mode with energy $T_{H}$ supported below $r=R+2 G T_{H}$ in terms of quantum field theory on a fixed spacetime containing a black hole of radius $R$ : if we add a particle to such a mode, then considering both the particle and the black hole we find a total energy $R / 2 G+T_{H}$ concentrated in a region smaller than the corresponding Schwarzschild radius! Thus, if the particle's energy were spherically distributed, one would expect that such a state is better described by a larger black hole (of size $R+2 G T_{H}$ ) than by an excitation of the original black hole (of size $R$ ). One therefore expects that fluctuations in the occupation numbers of such modes are more properly described as excitations of the horizon, and therefore that their contributions to the entropy are already included in $S_{B H}$. As a result, if one is considering that part of the thermal atmosphere ${ }^{4}$ which acts as a correction to $S_{B H}$, then one should impose a cutoff at some $r_{c}>R+2 G T_{H}$. Since $T_{H} \sim 1 / R$ and since for $r \ll R$ the proper distance $L$ from the horizon satisfies $L^{2} / R \sim r-R$, one indeed arrives at the conclusion that the cutoff must be at least a proper distance of order $L_{c} \sim \ell_{p}$ from the horizon.

On the other hand, as discussed above there are many modes near the horizon and, in calculating the entropy of the thermal atmosphere we have considered all of these modes to be thermally excited. In particular, even for modes at a given scale $L$ from the horizon, there are $R^{2} / L^{2}$ such modes due to the size of the corresponding sphere around the black hole. Thus, a more careful consideration is in order, and it is to this task that we next turn. Note that it is necessarily fluctuations of the occupations numbers that are relevant as the expectation value of the stress-energy tensor remains small even very close to the horizon, with the expected energy density being of order $R^{-4}$. Thus, a shell of thickness $\ell_{p}$ around the horizon on average contains only an energy of order $\left(R^{2} \ell_{p}\right) R^{-4}=\ell_{p} R^{-2}$. In contrast, we saw above that an energy of order $T_{H} \sim R^{-1}$ is required to move the horizon out to this radius. Thus, only departures from the mean can significantly increase the cutoff beyond the naive Planck scale estimate. Note also that energy fluctuations are largest for modes with $E \sim T_{H}$.

As noted above, the effect of a spherical shell of mass on the black hole horizon is easy to compute. However, a localized disturbance on a scale $L \ll R$ is far from spherically symmetric. One could, of course, average over a sphere's worth of fluctuations to estimate a typical fluctuation in the total mass of the black hole. Such an estimate is discussed in the appendix, but is vastly over-conservative as the averaging will partially cancel positive

\footnotetext{
${ }^{4}$ This wording has been used because the above observation is not necessarily in disagreement with the idea that at least a part of the thermal atmosphere's entropy is a dual description of $S_{B H}$. On the other hand, it is not obviously identical. In particular, it does not suffer from the issues discussed in [8].
} 


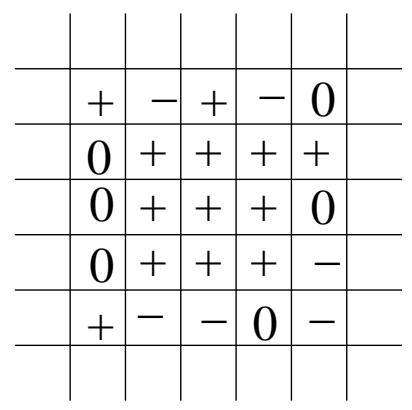

Figure 2: The central fluctuation $(+)$ happens to be surrounded by other fluctuations $(+)$ of the same sign. Fluctuations of the opposite sign (-) may occur farther away, but the central fluctuation will not feel their influence on a timescale $L$.

energy fluctuations on one side of the black hole against negative energy fluctuations on the opposite side of the black hole. Thus, it will dramatically underestimate the local fluctuations in the horizon location. In other words, it is clear that the largest effect comes from changes in the shape of the horizon as opposed to just the overall size.

How then shall we estimate this more localized effect on the horizon? Consider a positive energy fluctuation of length scale $L$ at a corresponding separation from the classical horizon. If this induces a bulge on the horizon which is large enough to capture the fluctuation itself, then it is clear that this must occur on a timescale ${ }^{5} L$. Note that this is also the natural lifetime of the fluctuation. Consider now the center of the fluctuation. On a timescale $L$ the center can receive no information from farther away than $L$. As a result, it cannot know whether it is indeed part of a homogeneous spherical shell of such fluctuations, or whether it is merely surrounded by an additional layer or so of similar fluctuations $^{6}$ (see figure 2). Thus, under reasonably common conditions, we should get the right answer (as to whether the horizion bulges outward and engulfs our fluctuation) by supposing that the black hole is in fact surrounded by a spherical shell of such fluctuations and determining whether this shell would add enough mass to the black hole to enlarge the horizon beyond the location of the fluctuations. Note that the shell has thickness $L$ (see figure 3); luckily, the calculation is just as easy for thick shells as for thin.

Let us consider a general spherically symmetric static metric of the form

$$
d s^{2}=-g_{t t}(r) d t^{2}+g_{r r}(r) d r^{2}+r^{2} d \Omega_{d-2}^{2},
$$

where as usual $d \Omega_{d-2}^{2}$ is the metric on the unit $(d-2)$-sphere. We take $f$ to have a first-order zero at $r=R$, representing the non-degenerate black hole horizon.

In a sufficiently smal region close to the horizon, we may approximate the metric in the $r, t$ directions by the standard Rindler metric:

\footnotetext{
${ }^{5}$ Say, as measured by freely falling observers initially at rest with respect to the black hole. Since we are primarily concerned with the perturbative regime, we may use the metric of the original Black Hole to compute times to leading order.

${ }^{6}$ It may just barely be able to tell whether the neighboring fluctuations have the same sign, but such a clumping will occur a frequency which is not parametrically small, and thus is large enough for our purposes.
} 


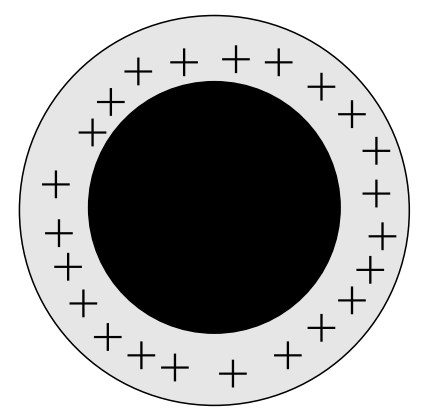

Figure 3: A shell of fluctuations $(+)$ around the black hole. Since each fluctuation is of size $L$, a given fluctuation cannot detect the others on a timescale less than $L$.

$$
d s^{2}=-\kappa^{2} \xi^{2} d t^{2}+d \xi^{2}+R^{2} d \Omega_{d-2}^{2}+\text { higher order in } \xi,
$$

where the constant $\kappa$ is the surface gravity, and is chosen so that $t$ is properly normalized; e.g., so that $\left|\partial_{t}\right| \rightarrow 1$ at infinity if the spacetime is asymptotically flat. The Hawking temperature $T_{H}$ is then $T_{H} \sim \kappa$ since we set $\hbar=1$. Note that for Schwarzschild black holes the description (3.2) will be valid whenever $r \ll R$, but for nearly extreme black holes we must approach the horizon more closely than the difference in radius between the outer and inner horizons. We will always assume that the fluctuations are close enough to the horizon for (3.2) to apply ${ }^{7}$.

Let us ask how far the horizon moves outward when we add a mass $\Delta m$ to the black hole but maintain spherical symmetry. The new horizon is where $g_{t t}$ now vanishes. Since it used to vanish at $r=R$, we set

$$
0=\Delta g_{t t} \approx \partial_{m} g_{t t} \Delta m+\partial_{r} g_{t t}(r-R)
$$

On the other hand, comparing (3.1) and (3.2), we see that $g_{t t} \sim-T_{H}^{2} \xi^{2}$, so we find

$$
\partial_{r} g_{t t} \sim-2 \xi T_{H}^{2} \partial_{r} \xi=-2 \xi T_{H}^{2} \sqrt{g_{r r}} .
$$

Since $g_{t t}$ vanishes only to first order, we see that $g_{r r}=\frac{c^{2}}{\xi^{2}}(1+O(\xi))$ for some constant $c$. Thus $r-R \sim \xi^{2} / 2 c$. Putting this together with (3.3) and (3.4), one finds that adding a mass $\Delta m$ moves the horizon to a new value of $r$ which, in the original metric, was located at a proper distance

$$
\xi=\sqrt{\frac{\partial_{m} g_{t t}}{T_{H}^{2}} \Delta m}
$$

from the original horizon.

Unfortunately, it seems that no general theorems concerning the form of $\partial_{m} g_{t t}$ are available. However, for the Schwarzschild and Reissner-Nordstrom solutions in $d$ spacetime dimensions one finds $\partial_{m} g_{t t}=($ const $) \ell_{p}^{d-2} r^{-(d-3)}$ where the derivative is taken holding all

\footnotetext{
${ }^{7}$ Any cut-off we find will be parametrically small in the Planck length, so only for black holes parametrically close to extremality can this approximation fail.
} 
charges constant ${ }^{8}$ and const is a number that depends at most on the dimension; i.e., which is independent of any charges or cosmological constant. We will therefore assume that any new black hole considered follows this pattern.

With this understanding, one finds that the additional mass required to, roughly speaking, "move the horizon outward a proper distance $\xi$ " is given by

$$
\Delta m_{\text {sphere }} \sim \frac{T_{H}^{2} R^{d-3} \xi^{2}}{\ell_{p}^{d-2}} .
$$

The subscript sphere has been added to remind us that we have considered only spherically symmetric configurations above. For example, the outcome above might be attained by adding a spherical shell of mass $\Delta m_{\text {sphere }}$ to our original black hole.

We now return to considering a single fluctuation of physical size $L$ located at a proper distance $L$ from the classical horizon of our black hole. We have seen that the typical energy of this fluctuation is $\Delta m_{\text {fluct }} \sim T_{H}$, so that a spherical shell of such fluctuations (as shown in figure 3) would have a mass $\Delta m_{\text {sphere }} \sim T_{H}(R / L)^{d-2}$. Comparison with (3.6) then shows that $L>\xi$ if and only if $L>L_{c}$ where

$$
L_{c}^{d} \sim \frac{R}{T_{H}} \ell_{p}^{d-2} .
$$

Putting together our logic above, this $L_{c}$ represents a rough measure of the quantum width of the horizon and fluctuations within $L_{c}$ of the classical horizon cannot be cleanly separated from the black hole itself.

In general, there will be $N$ propagating degrees of freedom (i.e., helicity states of quantum fields) at the scale $L_{c}$. Any combination of bosons and fermions leads to a typical increase in the energy $\Delta m_{\text {fluct }}$ of fluctuations in a given region by a factor of $\sqrt{N}$, so the cut-off length $L_{c}$ becomes

$$
L_{c}^{d} \sim \frac{R}{T_{H}} N^{1 / 2} \ell_{p}^{d-2}
$$

The entropy of the thermal atmosphere down to this cut-off is then $S \sim N A / L_{c}^{d-2}$; i.e., smaller than $S_{B H} \sim A / \ell_{p}^{d-2}$ unless $N$ is parametrically large in $\left(R / \ell_{p}\right)$. Note that $N$ should include any effective field with a mass $m \lesssim 1 / L$, so that in general $N$ depends on $L$ and (3.8) represents only an implicit solution for the cut-off scale.

\section{Discussion}

In the above sections we have argued that the horizon of any black hole has an effective "quantum width" given by (3.8). This supports Sorkin's suggestion 13] that fluctuations of the horizon provide a cut-off on the entropy of the black hole's thermal atmosphere, suppressing this entropy parametrically in comparison with the black hole's BekensteinHawking entropy. Our work provides a fully relativistic treatment and includes both uncharged black holes and those near the extremal limit, in particular giving an explicit

\footnotetext{
${ }^{8}$ This variation models fluctuations in uncharged fields, such as the metric itself. One could of course also consider fluctuations of charged fields.
} 
dependence of the cut-off on the Hawking temperature separate from the dependence on the size $R$ of the black hole. Our arguments concern general spherical black holes with non-degenerate (i.e., non-extreme) horizons, but an extension to the rotating case and a direct computation for extreme black holes would clearly be of interest. For Schwarzschild black holes in $3+1$ dimensions, our estimate of the width $L_{c}$ is only just below nuclear length scales for astrophysical black holes and begins to approach atomic length scales for the largest known supermassive black holes.

Our estimate of the width is based on the conjecture that whether the horizon bulges outward to engulf a particular fluctuation depends at most on whether the first few layers of surrounding fluctuations have a similar sign (as in figure 2), and does not depend on the presence of fluctuations farther away. In this case, we may model the calculation by assuming that there are in fact a sphere's worth of such fluctuations and use simple results for spherically symmetric perturbations of the black hole. As discussed in the Appendix, a skeptic could raise some questions about this conjecture, and a complete proof or counterexample would be much desired. The question should be ammenable to study by various perturbative methods, and we look forward to future results in this direction ${ }^{9}$.

In the absence of such precise results, we may also take comfort from the more conservative estimate given in the appendix. This estimate considers only the average fluctuation in the mass of a spherical shell and so does not rely on any conjectures. On the other hand, it yields the desired result only for $d \leq 5$.

Let us, however, proceed with the estimate (3.8). One interesting feature is that the entropy of the thermal atmosphere continues to give a large contribution in the presence of a sufficiently large number $N$ of propagating fields. Under such circumstances the black hole will rapidly decay into a fireball of thermal radiation, unless the black hole is close to extremality so that its temperature is low (in which case the thermal atmosphere can be fully excited with only a small fraction of the black hole's energy).

On a related note, we have seen that even for $N=1$ a large black hole allows of order $\left(R / L_{c}\right)^{d-2}$ modes to be excited with an energy of order $T_{H}$. Consider now a particle placed in a mixed state, which occupies an undetermined member of this set of modes. The von-Neumann entropy of the corresponding density matrix is then of order $\ln \left(R / L_{c}\right)$, which is parametrically larger than $E / T_{H} \sim 1$. The reader might at first wonder what will happen when such a particle falls into the black hole (since the black hole's entropy will increase only by $E / T_{H}$ by the first law).

Indeed, following the logic of [1], one might expect it to lead to a violation of the generalized second law (GSL). However, as discussed in my talk at the conference (and as described in [5, 6, 7]) other, perhaps unexpected, effects will intervene to save the GSL. Since the particle's energy is $T_{H}$, it is clear that external observers will describe the particle as being added to a rather busy thermal state, and not just to the vacuum. Such observers will be most concerned with the change in entropy they assign to the process by which one particle falls into the black hole but a thermal state remains outside. Note that since the thermal state is well-occupied, the fundamental indistinguishability of particles may

\footnotetext{
${ }^{9}$ It would appear that the closest result in the existing literature is provided by [17], which considers two black holes of equal mass as opposed to a single black hole with a small perturbation.
} 
come into play and one cannot guess the answer from a simple model of distinguishable particles. In fact, as discussed in [7], an ensemble of distinguishable particles which allows fluctuations in particle number fails to be well-defined in our present circumstance.

As follows from the corresponding analysis in [7], this change in entropy does not in fact grow arbitrarily large with $R / L$ but instead asymptotes to $E / T_{H}$, a value that preserves the second law ${ }^{10}$. It is only observers who fall across the horizon which assign the particle an entropy of order $\ln (R / L)$, but such observers do not see the particle disappear and face no issues with regard to the GSL.

On the other hand, one can ask interesting questions about how the experiences of such observers could be consistent with a description of the black hole interior in terms of a Hilbert space with a finite number $e_{B H}^{S}$ of dimensions. We shall not discuss such questions in detail here, as readers will no doubt interpret the outcome according to their pre-existing views of black hole entropy. Some readers will see evidence that $S_{B H}$ counts only "the ways the black hole can interact with the external universe" and not the full set of interior states, while others will see manifestations of "complementarity" between various observers inside the black hole. The latter might be protected by limitations on the transfer of information between such observers analogous to those discussed in [19] for observers comparing Hawking radiation with the original objects falling into a black hole.

Returning now to somewhat firmer ground, perhaps the most interesting observation regarding our result (3.8) is the discrepancy with naive expectations based on a 'stretched horizon' picture of the black hole. The stretched horizon is typically described as a membrane just outside the black hole's classical horizon having properties that make its classical dynamics indistinguishable from the black hole itself [21]. Various differing proposals have been made for the details to incorporate quantum effects; for example, [19, 20] place the stretched horizon on the symmetry sphere with $\ell_{p}^{2}$ more area than the classical horizon while [22] chooses the sphere with string-scale temperature, and [19, 23] choose the Planck temperature sphere. However, at least for Schwarzschild black holes, a membrane of Planck tension and Planck temperature (and thus located at roughly a Planck scale proper distance from the classical horizon) naturally reproduces the thermodynamics (e.g., energy and entropy) of the black hole and so seems to be preferred. On the other hand, since there are no infrared divergences in $2+1$ or higher dimensions, typical fluctuations in the location of such a membrane (corresponding to black holes in $d \geq 4$ ) would also tend to be of intrinsically Planck scale, and thus would be parametrically smaller than our $L_{c}$. This suggests that the stretched horizon concept may be in need of refinement; a process that may provide fertile ground for future research.

Acknowledgments: The author would like to thank Raphael Bousso, Ram Brustein, Chris Herzog, Gary Horowitz, Stefan Hollands, Dan Kabat, Luis Lehner, David Lowe, Mark Spradlin, and the participants of the Adriatic 2003 conference for interesting discussions. Special thanks go to Djordje Minic, Simon Ross, and Rafael Sorkin for many discussions

\footnotetext{
${ }^{10}$ A closely related phenomenon was discovered in [18], which observed that a background of Unruh radiation downgrades the fidelity of quantum teleportation, indicating a loss of access to quantum information by observers who do not cross the horizon.
} 
during our collaborations and to Gary Horowitz and Eric Poisson for help in locating reference [17]. This work was supported in part by NSF grants PHY00-98747, PHY9907949, and PHY03-42749, and by funds from the University of California.

\section{A. An overly conservative estimate}

In section 3, we argued that one could estimate local distortions of the horizon caused by energy fluctuations by considering a spherical shell of such fluctuations surrounding the black hole. The idea was that, due to the short timescales involved, whether or not a given fluctuation was engulfed by a distortion of the horizon should be independent of the existence of neighboring fluctuations. However, the argument clearly falls short of a rigorous proof and must therefore be termed a conjecture. In particular, a skeptic might worry that the presence or absence of neighboring fluctuations over a time in the past of order $R$ could affect the initial conditions in an important way, invalidating the arguments of section 3 .

Ultimately, this should be settled by a complete calculation. However, not having such a calculation at hand, it is also of interest to pursue other models of horizon fluctuations not based on the conjecture above. To this end, let us now consider the effect of averaging the fluctuations over a sphere around the black hole. This is clearly overly conservative, as it averages independent positive and negative fluctuations into an overall expansion or contraction of the black hole horizon. However, it provides a similar qualitative behavior to (3.8) in spacetime dimensions $d \leq 5$ and, in particular, reproduces exactly Sorkin's Newtonian result in $d=4$.

The idea is a simple extension of our analysis so far. We simply note that there are of order $N(R / L)^{d-2}$ independent fluctuations of size $L$ around the sphere. As a result, a typical fluctuation in the mass contained in this thick shell is of order $\Delta m_{\text {fluct }} \sqrt{N(R / L)^{d-2}}=$ $\frac{T_{H} N^{1 / 2} R^{(d-2) / 2}}{L^{(d-2) / 2}}$. Comparing this with $(3.5)$ yields

$$
L_{c}^{(d+2) / 2} \sim \frac{N^{1 / 2} \ell_{p}^{d-2}}{T_{H} R^{(d-4) / 2}} .
$$

In particular, for a Schwarzschild black hole in $d=4$ one finds

$$
L_{c} \gtrsim N^{1 / 6}\left(R \ell_{p}^{2}\right)^{1 / 3},
$$

and again this cutoff leads to negligible entropy in the thermal atmosphere. However, since $T_{H}$ generally scales with $1 / R$, we see that (A.1) fails to suppress the thermal atmosphere's entropy relative to the Bekenstein-Hawking entropy in $d \geq 6$ and that more care will be needed for such cases.

\section{References}

[1] J. D. Bekenstein, "Black Holes And Entropy," Phys. Rev. D 7, 2333 (1973); J. D. Bekenstein, "Generalized Second Law Of Thermodynamics In Black Hole Physics," Phys. Rev. D 9, 3292 (1974). 
[2] J. D. Bekenstein, "Quantum information and quantum black holes," arXiv:gr-qc/0107049.

[3] L. Susskind, "The World as a hologram," J. Math. Phys. 36, 6377 (1995) [arXiv:hep-th/9409089].

[4] G. 't Hooft, "Dimensional Reduction In Quantum Gravity," arXiv:gr-qc/9310026.

[5] D. Marolf and R. Sorkin, "Perfect mirrors and the self-accelerating box paradox," Phys. Rev. D 66 (2002) 104004 [arXiv:hep-th/0201255].

[6] D. Marolf and R. Sorkin, "On the Status of Highly Entropic Objects", hep-th/0309218.

[7] D. Marolf, D. Minic and S. F. Ross, "Notes on spacetime thermodynamics and the observer-dependence of entropy," arXiv:hep-th/0310022.

[8] R. M. Wald, "The thermodynamics of black holes," Living Rev. Rel. 4, 6 (2001) [arXiv:gr-qc/9912119].

[9] G. 't Hooft, "On The Quantum Structure Of A Black Hole," Nucl. Phys. B 256, 727 (1985).

[10] S. Mukohyama, "Aspects of black hole entropy," arXiv:gr-qc/9912103.

[11] R. Brustein and A. Yarom, "Thermodynamics and area in Minkowski space: Heat capacity of entanglement," arXiv:hep-th/0311029.

[12] R. D. Sorkin, "On The Entropy Of The Vacuum Outside A Horizon," Gen. Rel. Grav., proceedings of the GR10 Conference, Padova 1983, ed. B. Bertotti, F. de Felice, A. Pascolini (Consiglio Nazionale della Ricerche, Roma, 1983) Vol. 2; L. Bombelli, R. K. Koul, J. H. Lee and R. D. Sorkin, "A Quantum Source Of Entropy For Black Holes," Phys. Rev. D 34, 373 (1986); M. Srednicki, "Entropy and area," Phys. Rev. Lett. 71, 666 (1993) [arXiv:hep-th/9303048].

[13] R. D. Sorkin, "How wrinkled is the surface of a black hole?," arXiv:gr-qc/9701056.

[14] G. Lopes Cardoso, B. de Wit and T. Mohaupt, "Corrections to macroscopic supersymmetric black-hole entropy," Phys. Lett. B 451, 309 (1999) [arXiv:hep-th/9812082]; G. Lopes Cardoso, B. de Wit and T. Mohaupt, "Deviations from the area law for supersymmetric black holes," Fortsch. Phys. 48, 49 (2000) [arXiv:hep-th/9904005]; G. Lopes Cardoso, B. de Wit and T. Mohaupt, "Area law corrections from state counting and supergravity," Class. Quant. Grav. 17, 1007 (2000) [arXiv:hep-th/9910179].

[15] C. Misner, K. Thorne, and J. Wheeler, Gravitation (W.H. Freeman and Co., New York, 1970).

[16] J. M. Maldacena, "The large N limit of superconformal field theories and supergravity," Adv. Theor. Math. Phys. 2, 231 (1998) [Int. J. Theor. Phys. 38, 1113 (1999)] [arXiv:hep-th/9711200]. O. Aharony, S. S. Gubser, J. M. Maldacena, H. Ooguri and Y. Oz, "Large N field theories, string theory and gravity," Phys. Rept. 323, 183 (2000) [arXiv:hep-th/9905111].

[17] G. B. Cook and A. M. Abrahams, "Horizon Structure Of Initial Data Sets For Axisymmetric Two Black Hole Collisions," Phys. Rev. D 46, 702 (1992).

[18] P. M. Alsing, D. McMahon and G. J. Milburn, "Teleportation in a non-inertial frame," arXiv:quant-ph/0311096.; P. M. Alsing and G. J. Milburn, "Teleportation with a uniformly accelerated partner," Phys. Rev. Lett. 91, 180404 (2003) [arXiv:quant-ph/0302179]. 
[19] L. Susskind, L. Thorlacius, and J. Ugglum, "The stretched horizon and black hole complementarity", Phys. Rev. D 48, 3743 (1993) [arXiv:hep-th/9306069].

[20] L. Susskind and L. Thorlacius, "Gedanken experiments involving black holes," Phys. Rev. D 49, 966 (1994) [arXiv:hep-th/9308100].

[21] K. S. Thorne, R.H. Price, and D. A. Macdonald, Black Holes: The Membrane Paragdigm, (Yale University Press, 1986).

[22] A. Sen, "Extremal black holes and elementary string states", Mod. Phys. Lett. A 10, 2081 (1995) [arXiv:hep-th/9204099].

[23] N. Ilizuka, D. Kabar, G. Lifschytz, and D. A. Lowe, "Stretched Horizons, Quasiparticles, and Quasinormal modes," Phys. Rev. D 68084021 (2003) [arXiv:hep-th/0306209]; N. Ilizuka, D. Kabar, G. Lifschytz, and D. A. Lowe, "Quasiparticle picture of black holes and the entropy area relation," Phys. Rev. D 67124001 (2003) [arXiv:hep-th/0212246]. 